\title{
JOIN-PRINCIPAL ELEMENT LATTICES
}

E. W. JOHNSON

\begin{abstract}
Let $(e, M)$ be a local Noether lattice. If the maximal element $M$ is meet principal, it is well known and easily seen that every element of $\mathscr{E}$ is meet principal. In this note, we obtain the corresponding result for $M$ joinprincipal. We also consider join-principal elements generally under the assumption of the weak union condition and show, for example, that the square of a join-principal element is principal.
\end{abstract}

Throughout we assume that $\mathcal{E}$ is a local Noether lattice with maximal element $M$.

THEOREM 1. If $A \in \mathcal{L}$ is join-principal, then, for each $n \geqslant 1, A^{n}$ has a unique minimal basis in $\mathcal{L} /((0: A) \wedge A)$.

Proof. Use Theorem 1 of [2] to choose a minimal base $E_{1}, \ldots, E_{k}$ for $A$ such that $E_{i} E_{j}=0$ whenever $i \neq j$.

Let $F$ be any principal element $\leqslant A^{n} \vee((0: A) \wedge A)$. Then by Lemma 1 of [3], there exist principal elements $F_{1}, \ldots, F_{k+1}$ such that

$$
F \vee\left(\bigvee_{i \neq j} F_{i} E_{i}^{n}\right)=\bigvee_{i} F_{i} E_{i}^{n}
$$

for $j=1, \ldots, k+1$, where $E_{k+1}=I$ and $F_{k+1} \leqslant(0: A) \wedge A$.

Then $F E_{j}=F_{j} E_{j}^{n+1}(1 \leqslant j \leqslant k)$, so

$$
F A=\underset{1 \leqslant j \leqslant k}{\bigvee} F_{j} E_{j}^{n+1}=\underset{1 \leqslant j \leqslant k}{\bigvee} F_{j} E_{j}^{n} A
$$

Hence

$$
F \vee(0: A)=\left(\underset{1 \leqslant j \leqslant k}{\vee} F_{j} E_{j}^{n}\right) \vee(0: A) .
$$

So, since principal elements are join-irreducible,

$$
F \vee((0: A) \wedge A)=F_{j} E_{j}^{n} \vee((0: A) \wedge A),
$$

for some $j$. Hence either $F \vee((0: A) \wedge A)=E_{j}^{n} \vee((0: A) \wedge A)$, or $F$ $\leqslant M A^{n} \vee((0: A) \wedge A)$ and $F$ cannot be used in a minimal base for $A^{n}$ in

Received by the editors February 25, 1974.

AMS (MOS) subject classifications (1970). Primary 06A20. 
$\mathcal{L} /(0: A) \wedge A$. It follows that the only minimal bases for $A^{n}$ in $\mathcal{L} /((0: A) \wedge A)$ are subsets of $E_{l}^{n} \vee((0: A) \wedge A), \ldots, E_{k}^{n} \vee((0: A) \wedge A)$. On the other hand, if $E_{j}^{n} \leqslant E_{1}^{n} \vee \cdots \vee \hat{E}_{j}^{n} \vee \cdots \vee E_{k}^{n} \vee((0: A) \wedge A)$, then $E_{j}^{n} A$ $=E_{j}^{n+1} \leqslant\left(E_{1}^{n} \vee \cdots \vee \hat{E}_{j}^{n} \vee \cdots \vee E_{k}^{n} \vee((0: A) \wedge A)\right) E_{j}=0$. Hence $A^{n}$ has the unique minimal base in $\mathcal{E} / A \wedge(0: A)$ consisting of those $E_{j}^{n}$ $\vee(A \wedge(0: A))$ with $E_{j}^{n} A \neq 0$. Q.E.D.

COROLlary 2. If $\mathcal{E}$ satisfies the weak union condition and if $A \in \mathcal{E}$ is joinprincipal, then $A=E \vee((0: A) \wedge A)$ for some principal element $E$. In particular, $A^{2}=E^{2}$ is principal.

Proof. If the weak union condition is satisfied, only principal elements have unique minimal bases. Since $E / A \wedge(0: A)$ inherits the hypothesis from $\varrho$, the result follows.

COROllary 3. Let $(R, M)$ be a local ring in which $M$ is join-principal. Then, as an $R$-module, $M$ is the direct sum of a cyclic $R$-module and an $R / M$-vector space.

In the general setting, we get the following

THEOREM 4. If the maximal element $M$ of $\mathcal{L}$ is join-principal, then $\mathcal{L} /(0: M)$ is distributive.

Proof. Let $F$ be any principal element of $\mathcal{E}, F \leqslant 0: M$. By Theorem 1 and Corollary 1 of [2], there exist principal elements $E_{1}, \ldots, E_{k}$ in $\mathcal{L}$ such that $E_{1} \vee(0: M), \ldots, E_{k} \vee(0: M)$ are an independent minimal base of $M$ in $\mathcal{L} /(0: M)$. Choose $n$ so that $F \leqslant M^{n} \vee(0: M)$ and $F \leqslant M^{n+1} \vee(0: M)$. Since $M$ is join-principal in $\varrho$, it follows that $F \vee(0: M)=E_{i}^{n} \vee(0: M)$ for some $i$. Since the $E_{j} \vee(0: M)$ are independent, it follows that $\mathcal{L} /(0: M)$ is distributive. Q.E.D.

COROllary 5. If the maximal element $M$ of $\mathcal{E}$ is join-principal, then every element of $\mathcal{L}$ is join-principal.

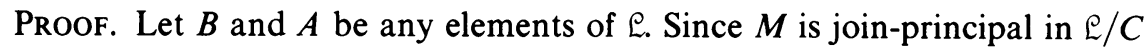
for every $C$, it suffices to show that $B A: A=B \vee(0: A)$.

Let $E_{1}, \ldots, E_{k}$ be independent principal elements such that $E_{1} \vee(0: M)$, $\ldots, E_{k} \vee(0: M)$ form the minimal base for $M$ in $\varrho / 0: M$. Then from the proof of Theorem 4, $A B: A$ and $B \vee(0: A)$ are both joins of powers of the $E_{i} \vee(0: M)$ in $\mathcal{L} / 0: M$.

Set $B \vee(0: A)=E_{1}^{s_{1}} \vee \cdots \vee E_{k}^{s_{k}} \vee(0: M)$, and assume $E_{i}^{n} \leqslant B A: A$. Then $E_{i}^{n} A \leqslant A(B \vee(0: A))=A E_{1}^{s_{1}} \vee \cdots \vee A E_{k}^{s_{k}}$. Since $E_{j} X$ is a power of $E_{j}$ for every $X \neq I$ and since the $E_{j}$ are independent, it follows that $E_{i}^{n} A \leqslant E_{i}^{s_{i}} A$. If $n<s_{i}$, then $A \leqslant E_{i}^{s_{i}-n} A \vee\left(0: E_{i}^{n}\right)$. However, in this case, $A \leqslant 0: E_{i}^{n}$ and $E_{i}^{n} \leqslant 0: A$. If $n \geqslant s_{i}$, then $E_{i}^{n} \leqslant E_{1}^{s_{1}} \vee \cdots \vee E_{k}^{s_{k}}$. In either case, $E_{i}^{n} \leqslant B \vee(0: A)$. Hence $B A: A \leqslant B \vee(0: A)$. Q.E.D.

\section{REFERENCES}

1. E. W. Johnson and J. P. Lediaev, Structure of Noether lattices into join-principal maximal 
elements, Pacific J. Math. 37 (1971), 101-108. MR 46 \#108.

2. Join-principal elements in Noether lattices, Proc. Amer. Math. Soc. 36 (1972), 73-78. MR 46 \#3301.

3. E. W. Johnson and Michael Detlefson, Prime sequences and distributivity in local Noether lattices, Fund. Math. 86 (1974), 149-156.

Department of Mathematics, University of Iowa, Iowa City, Iowa 52242 